\title{
Effect of treating severe nasal obstruction on the severity of obstructive sleep apnoea
}

\author{
H.A. McLean*, A.M. Urton*, H.S. Driver", A.K.W. Tan`, A.G. Day ${ }^{+}$, P.W. Munt* and M.F. Fitzpatrick*
}

\begin{abstract}
An association between mouth breathing during sleep and increased propensity for upper airway collapse is well documented, but the effect of treatment for nasal obstruction on mouth breathing during sleep and simultaneous obstructive sleep apnoea (OSA) severity has not been described previously.

A randomised single blind placebo- and sham-controlled crossover study of treatment (topical decongestant and external dilator strip) for nasal obstruction was carried out in 10 patients (nine males; mean \pm SEM $46 \pm 5$ yrs) with nasal obstruction and OSA. All patients had normal acoustic pharyngometry. The effect of treatment on nasal resistance, mouth breathing during sleep and OSA severity was quantified.
\end{abstract}

Treatment of nasal obstruction was associated with a dramatic and sustained reduction in nasal resistance and the oral fraction of ventilation during sleep (mean (95\% confidence interval) absolute reduction in oral fraction $30 \%(12-49)$ ). Improvements in sleep architecture were observed during active treatment, and there was a modest reduction in OSA severity (change in apnoea-hypopnoea index 12 (3-22)).

In conclusion, treating nasal obstruction reduced mouth breathing during sleep and obstructive sleep apnoea severity, but did not effectively alleviate obstructive sleep apnoea.

KEYWORDS: Mouth breathing, nasal obstruction, sleep apnoea, treatment

$\mathbf{U}$ nder normal circumstances, breathing during sleep is primarily nasal rather than oral [1], and considerable evidence has accumulated that nasal obstruction predisposes to obstructive sleep apnoea (OSA). Individuals describing nasal congestion due to allergy have shown a higher prevalence of OSA than those who do not [2]. Nasal resistance is an independent predictor of snoring severity [3], and is higher among snorers with OSA than among snorers without OSA [4]. Experimental occlusion of the nasal airway has been shown to induce sleep apnoea among normal subjects [58]. Similarly, seasonal allergic rhinitis is associated with an increased number of obstructive apnoeas during sleep [9].

However, despite this well-documented association, the effect of treating nasal obstruction on OSA severity has been highly variable. Mechanical nasal valvar splints have been studied in snoring [10], upper airway resistance syndrome [11] and OSA [12-18]. Although nasal splints were associated with a reduction in snoring frequency and/or intensity in three of these studies [10, 13, 16], the effect on sleep apnoea severity was variable and generally disappointing. Similarly, topical nasal decongestants did not reduce OSA severity in patients with nasal obstruction $[19,20]$, but the use of topical nasal steroids in patients with allergic rhinitis was associated with improved subjective sleep quality in adults [21] and with an improvement in apnoea-hypopnoea index (AHI) from 10.7 to 5.8 in children [22]. Nasal surgeries have also been used to treat OSA, but the results have been inconsistent and often lacked adequate objective outcome measurements [23-25]. However, SERIES et al. [26] demonstrated that the absence of pharyngeal narrowing (as measured by the posterior airway space) in patients with OSA delineated a group with a better response to nasal surgery.

In this study, the hypothesis was that relief of nasal obstruction in patients with OSA and a normal retroglossal airway would lead to a reduction in mouth breathing during sleep, and, hence, provide effective treatment for OSA syndrome.

\section{METHODS}

Design

A randomised placebo-controlled single blind crossover design was employed. Patients underwent two overnight polysomnograms separated
AFFILIATIONS

Depts of *Medicine, and

`Otolaryngology, Queen's University, and

\#Sleep Laboratory, and

${ }^{+}$Research Development Office, Kingston General Hospital, Kingston, ON, Canada.

CORRESPONDENCE

M.F. Fitzpatrick

Division of Respiratory and Critical

Care Medicine

Queen's University

102 Stuart Street

Kingston

ON, K7L 3N6

Canada

Fax: 16135491459

E-mail:mf19@post.queensu.ca

Received

April 172004

Accepted after revision:

October 082004

\section{SUPPORT STATEMENT}

This work was funded by grants from the Ontario Thoracic Society

(Toronto, ON, Canada), the

Physicians' Services Incorporated Foundation (Toronto, ON, Canada), and the William M. Spear Foundation (Queen's University, Kingston, ON Canada). 
by 1 night at home. On the active treatment night, patients received both a topical nasal decongestant $(0.4 \mathrm{~mL}$ oxymetazoline $0.05 \%$ in each nostril $1 \mathrm{~h}$ before lights out and again after $4 \mathrm{~h}$ ) and an external nasal-valve dilator strip (Breathe Right; CNS, Minneapolis, MN, USA). On the placebo night, patients received $0.4 \mathrm{~mL}$ sodium chloride $0.9 \%$ and a sham dilator strip (identical tape to the Breathe Right strip, but without the plastic core). Randomisation was conducted using a computergenerated table of random numbers. The technologist scoring the overnight sleep study was blinded to the treatment administered (the protocol included blinding of patients, but most became aware of the active treatment through an obvious reduction in nasal obstruction).

Nasal resistance (posterior active rhinometry [1]) was measured in erect and supine positions at the start and end of each overnight study.

Full polysomnography was conducted from 00:00 h until $07: 00 \mathrm{~h}$, or until the subject requested that the study be terminated. Respiratory events were scored using standard criteria [27].

Oral and nasal ventilation were simultaneously measured using a mask (7900 series; Hans Rudolph, Kansas City, MO, USA; dead space $31.3 \mathrm{~mL}$ ) with independent nasal and oral compartments, which was customised, as described previously [28], to eliminate any restriction to mouth opening. Identical pneumotachs (3700 series; Hans Rudolph; dead space $13.87 \mathrm{~mL}$ ) were inserted into the oral and nasal ports of the face mask. Each pneumotach was attached to a separate research pneumotach system (HSS100HR; Hans Rudolph) that provided a digital output ( $40 \mathrm{~Hz}$ sampling frequency) of nasal/oral breath-by-breath tidal volume, interfaced with the computerised polysomnographic montage. Air leaks, and communication between the oral and nasal compartments of the face mask were meticulously excluded as per the usual routine [1].

\section{Patients}

In total, 10 patients were studied (nine males; mean $\pm \mathrm{SD}$ (range) $46 \pm 5$ yrs (18-63); body mass index $27 \pm 1.5 \mathrm{~kg} \cdot \mathrm{m}^{-2}$ (23-37)). The inclusion criteria were as follows: 1) OSA syndrome [27] not yet receiving treatment; 2) clinically significant nasal obstruction (on the basis of clinical examination by M.F. Fitzpatrick) caused by turbinate mucosal enlargement with or without dynamic inspiratory collapse at the nasal valve; and 3) a normal retroglossal airway dimension at physical examination and acoustic pharyngometry [29]. Exclusion criteria included the presence of lung disease, or the use of medications known to alter nasal resistance.

\section{Statistical analysis}

Sleep apnoea severity (AHI) was analysed using the standard method for the two-period crossover study as described by FLEISS [30]. In addition to the primary test of treatment effect, tests were performed for period and ordering (differential carry-over) effects. The analysis of oral fraction during quiet sleep and during obstructive events was performed using a mixed model with random effects for subject and subject night. This method allowed the use of multiple measurements per subject night, while testing for the within-subject effects of body position, sleep stage and treatment.

\section{RESULTS}

All 10 patients completed the protocol; six were randomised to receive placebo/sham treatment first, followed by active treatment on the second night, and four patients to the reverse order. Baseline nasal resistance values (prior to administration of active or placebo/sham treatment) were markedly elevated prior to treatment on both study nights, but were not different between the placebo and active treatment nights in either the seated $(p=0.40)$ or supine $(p=0.30)$ positions (fig. $1 a$ and $b)$. Nasal resistance decreased profoundly with active treatment $(\mathrm{p}<0.01)$, and was maintained at or below $3 \mathrm{cmH} \mathrm{H}_{2} \mathrm{O} \cdot \mathrm{L}^{-1} \cdot \mathrm{s}^{-1}$ overnight, but did not change $(p=0.24)$ during the placebo night. Pre-treatment Modified Stanford Sleepiness Scale [31] scores were similar (active 2.1 \pm 0.5 , placebo/sham 2.9 \pm 0.5 ; $\mathrm{p}=0.2)$ on both nights.

\section{Sleep apnoea severity}

Statistical analysis revealed no significant order or period effects. The AHI fell by 12 on average ( $95 \%$ confidence interval (CI) 3-22) on the active treatment night as compared with the placebo/sham night $(\mathrm{p}<0.02$; fig. 2$)$. However, only one patient (whose AHI with placebo was 17 and with treatment
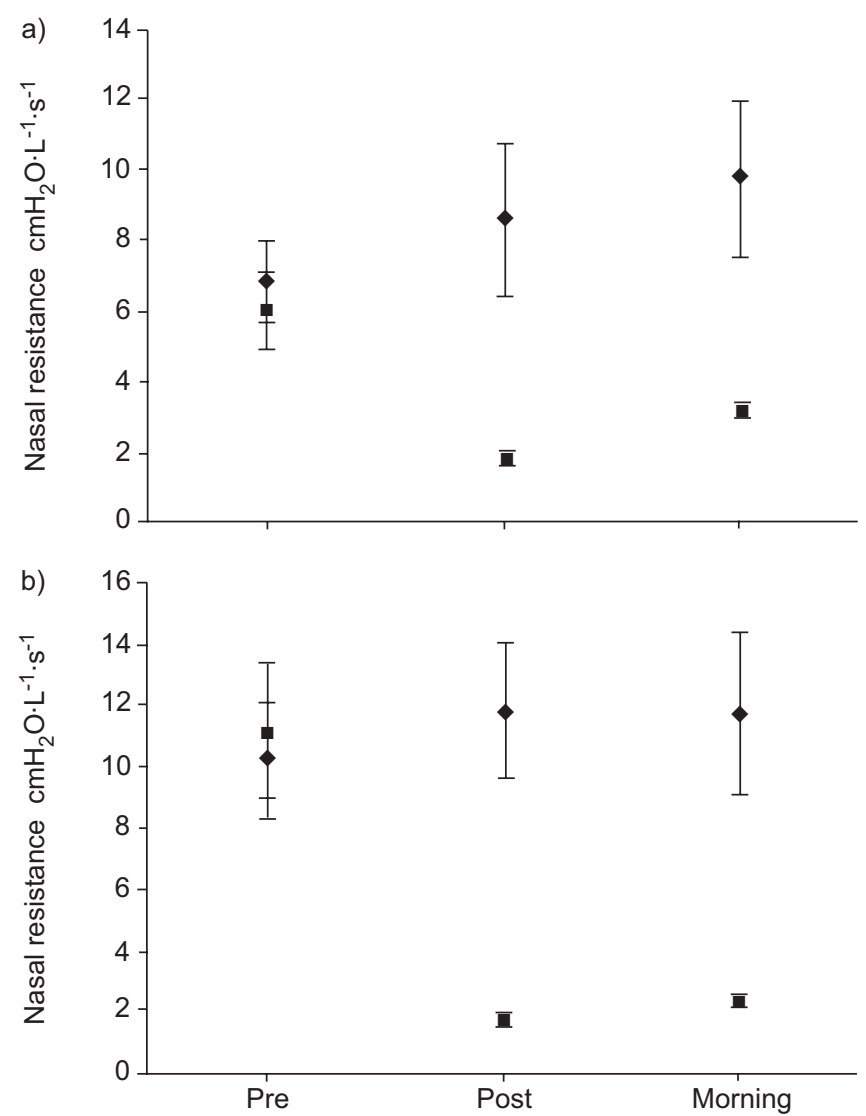

FIGURE 1. Nasal resistance values (mean \pm SEM) measured before treatment (pre), 30 min post-treatment (post) and at the end of the overnight recording (morning) in the seated position (a) and the supine (b) position. : sham/placebo; 口: active treatment. 


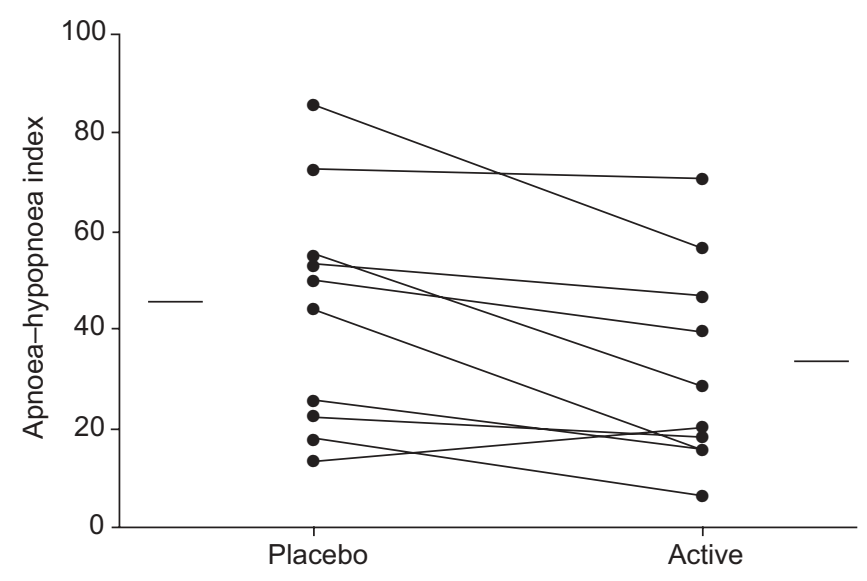

FIGURE 2. Effect of treatment for nasal obstruction on obstructive sleep apnoea severity. - : mean values. $\mathrm{p}<0.02$.

was 6) experienced a fall in $\mathrm{AHI}$ to $<15$ on the active treatment night. There was no significant correlation between the change in AHI from placebo/sham to active night, and the change in nasal resistance post-treatment from placebo/sham to active night measured in either the seated $\left(r^{2}=0.153 ; p=0.26\right)$ or supine $\left(r^{2}=0.001 ; p=0.92\right)$ positions. There were few respiratory events without associated arousals: the respiratory disturbance index (apnoeas or hypopnoeas terminating in arousal) on the placebo/sham night was $40 \pm 9$, and $30 \pm 4$ on the active treatment night $(\mathrm{p}=0.02)$.

\section{Mouth breathing during quiet sleep}

The oral fraction of inhaled ventilation during quiet breathing without obstructive events (fig. 3) was higher on the placebo night $(39 \pm 7 \%)$ than on the active treatment night $(8 \pm 6 \%)$. The $95 \%$ CI for the difference in oral fraction during quiet sleep between placebo/sham and active treatments was 12-49\% $(p=0.0043)$. No significant effect was observed between sleep stage (wake, rapid eye movement (REM), non-REM; $\mathrm{p}=0.23$ ) or body position (supine, lateral; $\mathrm{p}=0.70$ ) and the oral fraction during quiet sleep.

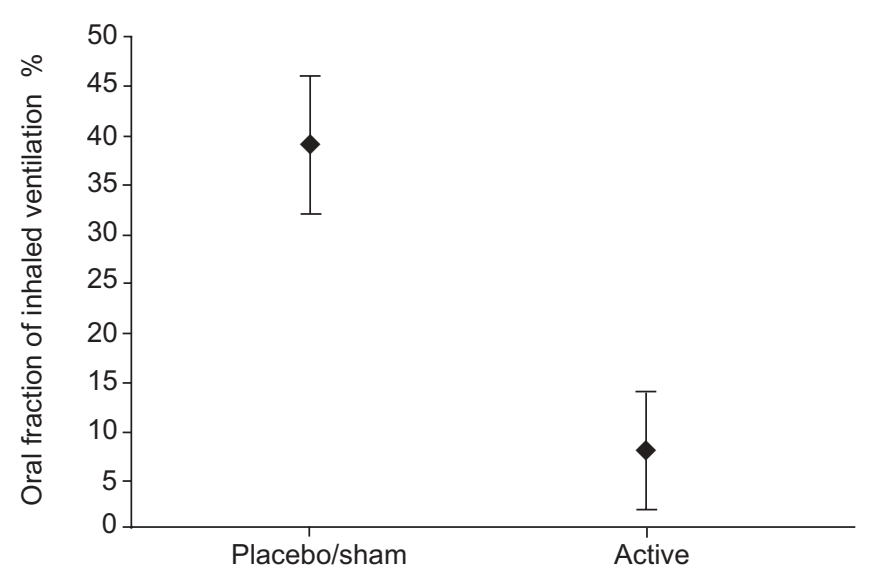

FIGURE 3. Effect of treatment for nasal obstruction on the oral fraction of inhaled ventilation during quiet sleep without obstructive events. Data are presented as mean \pm SEM. $p=0.004$

\section{Mouth breathing during obstructive events}

In order to assess the effect of active versus placebo/sham treatment on oronasal partitioning of ventilation during obstructive apnoeas and hypopnoeas, a random sample of 10 obstructive events that were standardised for position and sleep stage were analysed for each patient on each night, i.e. 200 events. The oral and nasal fractions were calculated breath by breath from the start of the event until the tidal volume (nasal and oral combined) fell below $50 \mathrm{~mL}$ or arousal occurred, and for the first three breaths after onset of the arousal (figs 4 and 5; table 1). When an obstructive event included an odd number of breaths, the first half of the event was extended to include the extra breath. Arousals in association with obstructive events were scored according to standard criteria [32]. For the purpose of the analysis, the arousal was considered to include the first three breaths occurring after an obstructive event and accompanied by electroencephalogram changes denoting wakefulness. Obstructive events were divided into four parts for analysis: "pre-event", "first half of event", "second half of event", and "arousal". The difference in the oral fraction of inhaled ventilation during the "pre-event" phase between the placebo/sham and active nights was mean (95\% CI) 2\% (10--7; $p=0.66)$. The oral fraction during the first half of the obstructive events was higher on the placebo/sham night than on the active treatment night (mean difference (95\% CI) 14\% $(2-27) ; \mathrm{p}=0.03)$. The difference in oral fraction during the second half of obstructive events between placebo/sham and active nights was highly variable and not statistically significant $(21 \%(-5-46) ; \mathrm{p}=0.1)$. Overall, combining both halves of the obstructive event, there was no significant difference in oral fraction between the two nights $(12 \%(-3-28) ; \mathrm{p}=0.10)$. The oral fraction at the time of arousal from obstructive events was significantly greater in the placebo/sham condition than in the active treatment condition (19\% (9-30); $\mathrm{p}=0.003)$.

The duration of obstructive events was not significantly different between the two treatment nights (placebo/sham night minus active treatment night $1.2 \mathrm{~s}(-2.5-4.8) ; \mathrm{p}=0.49)$.

\section{Sleep stage architecture}

Table 2 demonstrates the change in sleep-stage architecture with treatment. Despite the encumbrance of the oronasal mask and pneumotach system, significant improvements in sleep architecture (reduced stage 1 sleep, increased sleep efficiency, increased REM and slow-wave sleep) were observed with relief of nasal obstruction.

Modified Stanford Sleepiness Scale scores measured on the morning after each overnight sleep study were similar (active $2.7 \pm 0.1 ;$ placebo/sham $3.2 \pm 0.01 ; \mathrm{p}=0.2$ ).

\section{DISCUSSION}

This study demonstrates that relief of severe nasal obstruction in patients with a normal retroglossal airway is associated with a significant reduction in mouth breathing during sleep, improved sleep architecture and a modest improvement in OSA severity. The current study provides several new pieces of information, as follows.

1) This study included objective measurement of nasal resistance in the recumbent position, the usual sleeping 


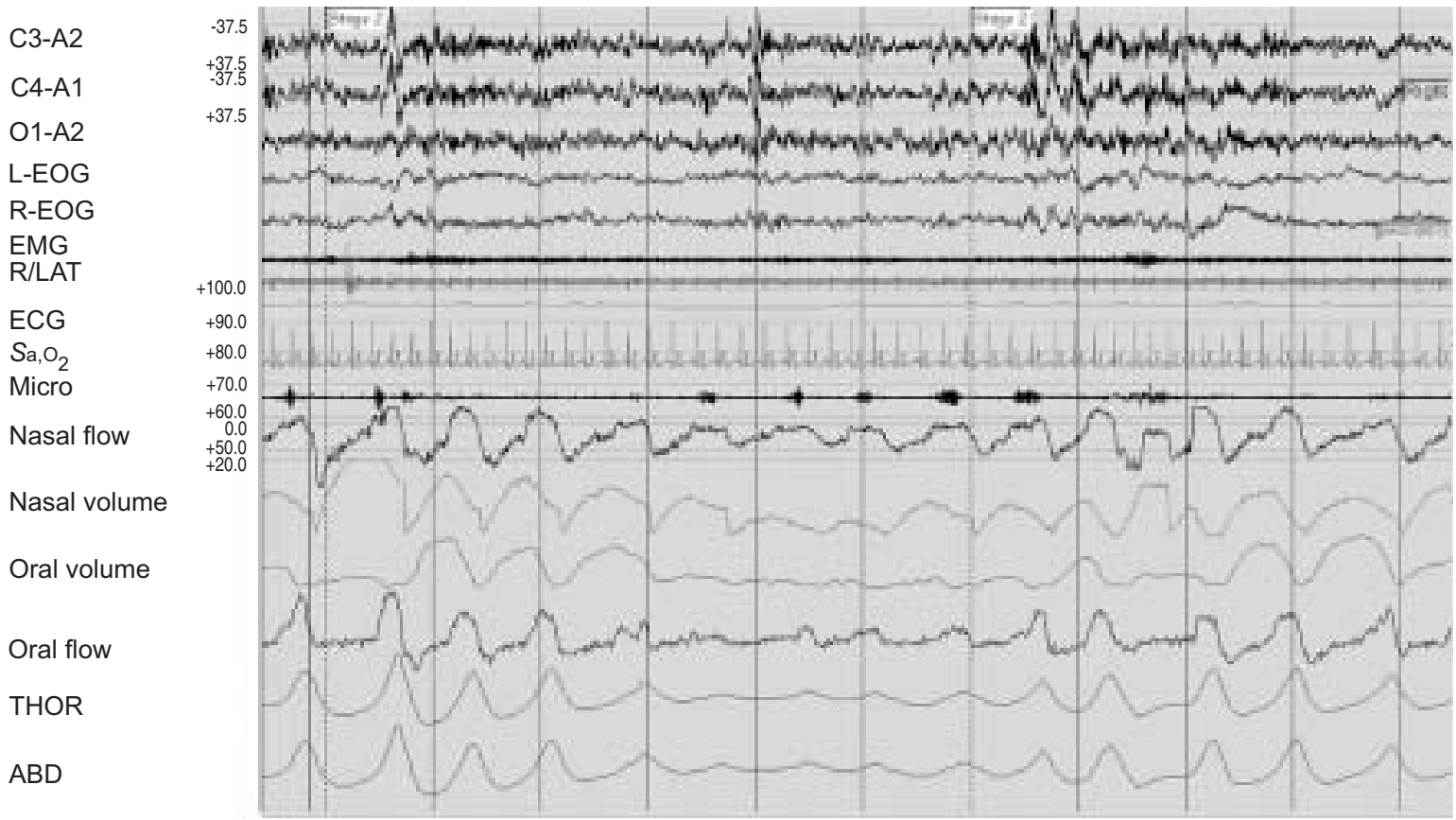

FIGURE 4. Active treatment night. An obstructive hypopnoea during $57 \mathrm{~s}$ of stage 2 sleep in the lateral position, demonstrating predominant nasal breathing (oral and nasal volume tracings) during the obstructive event. C3-A2, C4-A1, O1-A2: electroencephalogram electrode positions; L-EOG: left electro-oculogram; R-EOG: right electrooculogram; EMG: electromyogram; R/LAT: right and left anterior tibialis EMG; ECG: electrocardiogram; $\mathrm{Sa}_{1} \mathrm{O}_{2}$ : arterial oxygen saturation; Micro: snore vibration sensor; THOR: thoracic; ABD: abdominal.

C3-A2

C4-A1

O1-A2

L-EOG

R-EOG

EMG

R/LAT

ECG

$\mathrm{Sa}, \mathrm{O}_{2}$

Micro

Nasal flow

Nasal volume

Oral volume

Oral flow

THOR

$A B D$

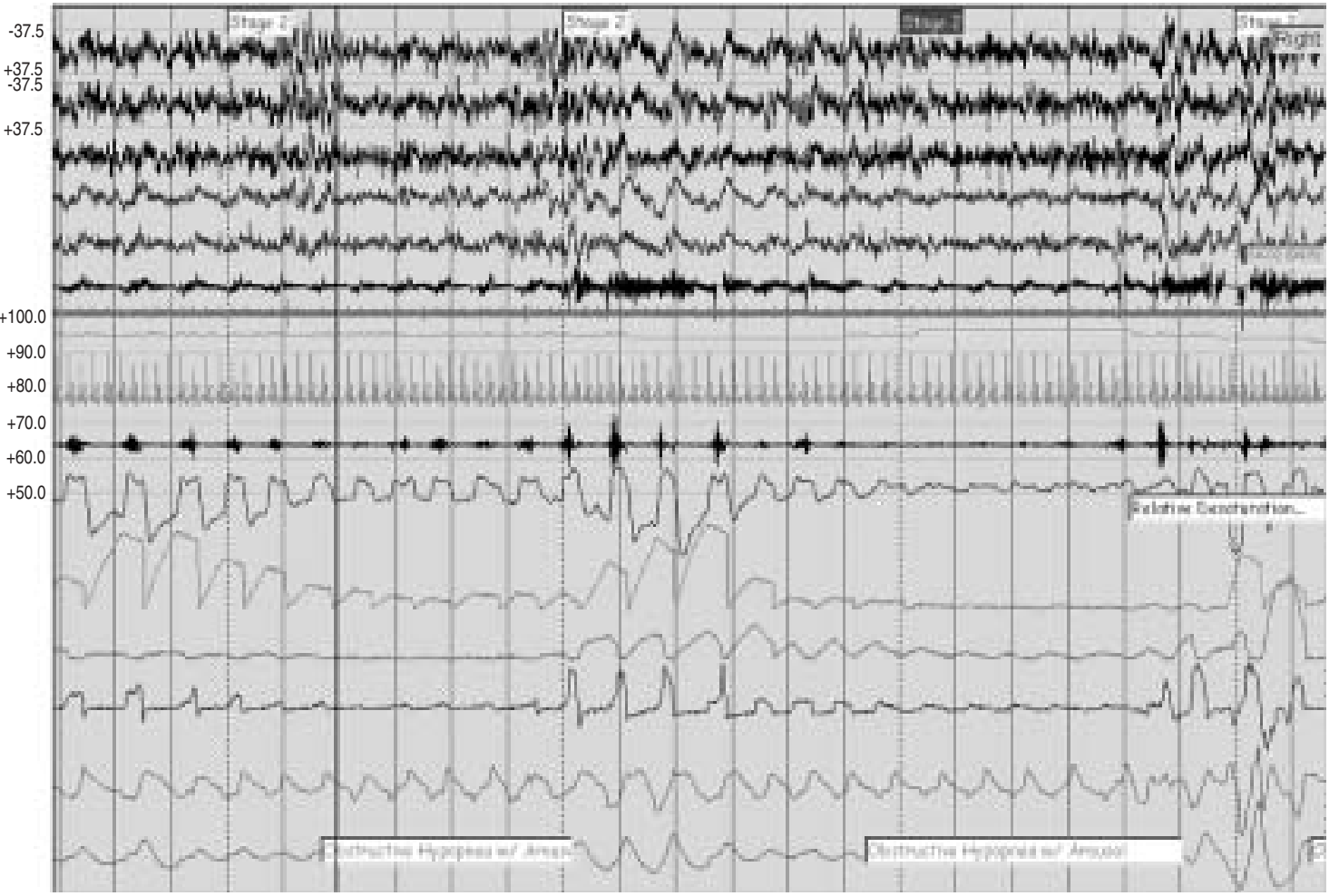

FIGURE 5. Placebo/sham night. Two obstructive events during 2 min of stage 2 sleep in the lateral position in the same patient as figure 4 . There is variability in oronasa ventilation between events (oral and nasal volume tracings). The first event demonstrates predominant nasal breathing with minimal oral breathing during the event, while the second event demonstrates predominance of oral breathing during the event. C3-A2, C4-A1, O1-A2: electroencephalogram electrode positions; L-EOG: left electrooculogram; R-EOG: right electro-oculogram; EMG: electromyogram; R/LAT: right and left anterior tibialis EMG; ECG: electrocardiogram; Sa, $\mathrm{O}_{2}$ : arterial oxygen saturation; Micro: snore vibration sensor; THOR: thoracic; ABD: abdominal. 


\begin{tabular}{lccc} 
TABLE 1 & $\begin{array}{l}\text { Effect of treatment for nasal obstruction on the oral } \\
\text { fraction of ventilation during different stages of } \\
\text { obstructive respiratory events }\end{array}$ \\
& Placebo/sham \% & Active \% & p-value \\
\hline Pre-event & $8.8 \pm 4$ & $7.2 \pm 4$ & 0.66 \\
First half of event & $48.7 \pm 12$ & $34.6 \pm 12$ & 0.03 \\
Second half of event & $49.3 \pm 13$ & $27.5 \pm 13$ & 0.08 \\
Arousal & $41.7 \pm 7$ & $22.4 \pm 7$ & 0.003 \\
\hline
\end{tabular}

Data are presented as mean $\pm \mathrm{SD}$, unless otherwise stated.

\begin{tabular}{lccc} 
TABLE 2 & $\begin{array}{l}\text { Effect of treatment for nasal obstruction on sleep } \\
\text { architecture }\end{array}$ \\
& Placebo & Active & p-value \\
\hline & 10 & 10 & \\
Subjects $\mathbf{n}$ & $24 \pm 4$ & $17 \pm 4$ & 0.02 \\
Stage 1 \% & $60 \pm 2$ & $53 \pm 2$ & 0.028 \\
Stage 2 \% & $8 \pm 2$ & $13 \pm 2$ & 0.028 \\
Stage 3 or 4 \% & $9 \pm 2$ & $16 \pm 2$ & 0.001 \\
REM \% & $78 \pm 4$ & $82 \pm 3$ & 0.001 \\
Sleep efficiency \% & $272 \pm 9$ & $294 \pm 14$ & 0.11 \\
Total sleep time min & $7.2 \pm 2$ & $6.6 \pm 2$ & 0.48 \\
Spontaneous arousal index & $132 \pm 36$ & $112 \pm 34$ & 0.60 \\
Supine min & \multicolumn{3}{l}{} \\
\hline
\end{tabular}

position, pre- and post-treatment of nasal obstruction in patients with OSA. This is important because nasal resistance increases significantly on moving from the erect to the recumbent position even in normal subjects, and the postural effect is further amplified in patients with pre-existing nasal obstruction [33, 34]. This aspect addresses a possible confounding factor in previous studies, i.e. that the increase in nasal resistance associated with recumbency could have offset the benefit of the treatment given to relieve nasal obstruction.

2) A single post-intervention measurement of nasal resistance has usually been reported as the evidence for efficacy of treatment for nasal obstruction in studies examining the effect of treatment of nasal obstruction on OSA severity. As OSA severity tends to increase over time during the night, independent of sleep stage or position, it was important to verify continuing efficacy of the active treatment for the duration of the overnight sleep study. In the current study, nasal resistance remained markedly elevated through the placebo night, but was reduced to within normal limits from $20 \mathrm{~min}$ post-treatment until the end of the overnight recording during the active treatment night. Hence, the modest nature of the improvement in OSA severity on active treatment could not be attributed to loss of the treatment effect over time on the active night.

3) This is the first time that oronasal partitioning of ventilation has been described during sleep, before and after relief of nasal obstruction. The current study demonstrates that nasal obstruction is associated with a greater proportion of mouth breathing at night, a finding that might seem intuitively obvious. However, it is remarkable that, even in the presence of very severe nasal obstruction in the current study, ventilation remained predominantly nasal in this group of patients; that is, patients continued to inspire through the high nasal resistance rather than bypassing it by switching to pure mouth breathing! This finding implies a major mechanical disadvantage of the oral breathing route in these patients and is consistent with the recent finding of a $\sim 2.5$-fold increase in upper airway resistance during sleep while mouth breathing as compared with nasal breathing in normal subjects [28]. Hence, in order to confer a mechanical advantage to pure mouth breathing during sleep, very severe (subtotal) nasal obstruction may be necessary.

4) The inclusion criteria in the current study were designed to select a patient group that the present authors, and others [26], felt might reasonably respond to relief of nasal obstruction as a treatment for OSA: these patients each had clinically important nasal obstruction, but did not have any evidence of anatomical compromise at the retroglossal level. The relatively poor response of OSA to normalising nasal resistance in this selected patient group is disappointing and provides powerful evidence against simple mechanical relief of nasal obstruction as a sole treatment for moderate or severe OSA, in the short term at least.

5) In the current study, the oral fraction fell from mean $39 \%$ during sleep in the placebo/sham condition to mean $8 \%$ (a level that is within normal limits [1]) with effective relief of nasal obstruction. This abrupt change in the oral fraction during sleep, in association with a fall in nasal resistance during a single night, implies that the degree of mouth breathing during sleep is determined primarily by the nasal resistance and not by behavioural factors. The patients in this study considered themselves to be predominantly mouth breathers during sleep, but on objective testing were actually predominantly nose breathers, despite having a higher than normal nasal resistance. This subjective judgment is notoriously inaccurate, even during wakefulness [35]. Hence, selfreports of mouth breathing during sleep are not a reliable indication of predominant mouth breathing.

Given the profound reduction in oral fraction observed with treatment, a greater reduction in OSA severity than that observed in the current study might have been expected. Interestingly, no trend towards a greater treatment effect in mild OSA was evident. The reduction in mouth breathing during sleep into the normal range in the current study, without a clinically important improvement in sleep apnoea severity, implies that mouth breathing per se may not be a critical factor in the presence or absence of OSA, but may simply be a surrogate marker for increased propensity to OSA.

Explanations for this relative disparity between improvement in the nasal airway and improvement in OSA severity, in the absence of corresponding objective data, are speculative. Certainly, in the current study, there was no clear systematic shift from mouth breathing to pure nasal breathing during obstructive events and, as figure 2 demonstrates, there was 
considerable within-patient variation in the breathing route from time to time, even within a given sleep stage and position. The current study did not measure jaw opening during sleep. It is possible that patients with chronic nasal obstruction could have greater habitual jaw opening during sleep, thus rendering the upper airway more collapsible [36], and that this habitual jaw posture during sleep might not revert to normal immediately after treatment of nasal obstruction. A disparity between mouth opening and mouth breathing has long been described, such that subjects with an open mouth may habitually breathe predominantly via the nose [35]. In such a scenario, it would be possible for individuals with nasal obstruction to reduce their oral ventilatory fraction in response to a reduction in nasal resistance, yet still suffer increased susceptibility to upper airway obstruction during sleep through persistent jaw opening. Indeed, patients with OSA have been shown to have greater jaw opening during sleep than normal subjects [37], and progressively greater jaw opening during obstructive sleep apnoeic events [38].

Another possible explanation for the disparity between reduction in mouth breathing and reduction in sleep apnoea severity is impaired function of nasal mucosal afferents in association with rhinitis, which might not recover immediately after simple relief of mucosal hyperaemia and oedema. Although nasal breathing consistently increases activation of the alae nasi, it is uncertain whether nasal afferents have any specific role in activation of the genioglossus or other pharyngeal dilator muscles [39, 40].

Patients in the current study experienced significant improvements in sleep architecture (improved sleep efficiency, less stage 1 sleep and more stage 2 sleep, slow-wave sleep and REM sleep) during the active treatment night as compared with the placebo/sham night, despite a relatively modest improvement in sleep apnoea severity with the active treatment. Others have also demonstrated a reduction in sleep fragmentation with treatment of nasal obstruction, even in the absence of any significant improvement in sleep apnoea severity [20, 25, 41]. Hence, the improvement in sleep architecture observed in the current study may relate more to a reduction in nasal discomfort with the active treatment rather than to improvement in sleep apnoea severity alone. An improvement in subjective sleep quality with relief of nasal obstruction, similar to that observed in the current study, has also been reported previously by other authors [21].

The current study has certain deficiencies. In particular, it is a short-term study: the effect of relieving nasal obstruction for a single night. It is possible that the longer-term effect of relieving nasal obstruction on OSA severity might be different. Having said that, it is difficult to discern any such trend in treatment effect with time, based on existing literature. VERSA and PIRSIG [42] have recently reviewed the outcome of nasal surgery as a treatment for OSA in nine studies (102 patients in total) that included pre-operative and post-operative measurement of AHI. The follow-up periods varied from 1 month to 44 months, and there was no evident trend to improved outcomes with longer follow-up.

As mentioned earlier, the current study did not include measurement of jaw position. Technically, jaw position could not have been measured in the current study using either of the previously published methods because of concern that an oral appliance [38] might alter oronasal distribution of ventilation and, specifically, that a strain gauge [37] might impose a restriction on mouth opening.

The inclusion criterion included a normal retroglossal airway dimension on acoustic pharyngometry. While this is a reproducible test [43] with available normative data [29], the measurements are made during wakefulness and in the erect seated position, and, therefore, do not reflect the physiological change observed with recumbency and sleep. Hence, individuals with a normal retroglossal dimension while awake may not be protected from retroglossal compromise while asleep.

The total sleep time in the current study was quite short, averaging only $4.5-5 \mathrm{~h}$. This was undoubtedly due to the rather cumbersome recording apparatus required to measure oral and nasal partitioning of ventilation during sleep, in addition to all of the other routine polysomnographic data. However, despite the relative brevity of sleep duration, it was fortunate that each patient had stages 1, 2, slow-wave sleep and REM sleep during both study nights, so that a valid comparison could be made between active treatment and the placebo/sham condition.

In conclusion, this study demonstrated that relief of nasal obstruction is associated with normalisation of mouth breathing during sleep, improved sleep architecture and a modest improvement in obstructive sleep apnoea severity. However, the improvement in obstructive sleep apnoea severity with relief of nasal obstruction was not sufficient to provide clinically effective treatment.

\section{ACKNOWLEDGEMENTS}

The authors acknowledge the technical assistance provided by M. San Pedro.

\section{REFERENCES}

1 Fitzpatrick MF, Driver HS, Chatha N, Voduc N, Girard AM. Partitioning of inhaled ventilation between the nasal and oral routes during sleep in normal subjects. J Appl Physiol 2003; 94: 883-890.

2 Young T, Finn L, Kim H. Nasal obstruction as a risk factor for sleep-disordered breathing. The University of Wisconsin Sleep and Respiratory Research Group. J Allergy Clin Immunol 1997; 99: S757-S762.

3 Metes A, Ohki M, Cole P, Haight JS, Hoffstein V. Snoring, apnea and nasal resistance in men and women. J Otolaryngol 1991; 20: 57-61.

4 Lofaso F, Coste A, d'Ortho MP, et al. Nasal obstruction as a risk factor for sleep apnoea syndrome. Eur Respir J 2000; 16 : 639-643.

5 Zwillich CW, Pickett C, Hanson FN, Weil JV. Disturbed sleep and prolonged apnea during nasal obstruction in normal men. Am Rev Respir Dis 1981; 124: 158-160.

6 Lavie P, Fischel N, Zomer J, Eliaschar I. The effects of partial and complete mechanical obstruction of the nasal passages on sleep structure and breathing in sleep. Acta Otolaryngol 1983; 95: 161-166. 
7 Suratt PM, Turner BL, Wilhoit SC. Effect of intranasal obstruction on breathing during sleep. Chest 1986; 90: 324-329.

8 Carskadon MA, Bearpark HM, Sharkey KM, et al. Effects of menopause and nasal occlusion on breathing during sleep. Am J Respir Crit Care Med 1997; 155: 205-210.

9 McNicholas WT, Tarlo S, Cole P, et al. Obstructive apneas during sleep in patients with seasonal allergic rhinitis. Am Rev Respir Dis 1982; 126: 625-628.

10 Todorova A, Schellenberg R, Hofmann HC, Dimpfel W. Effect of the external nasal dilator Breathe Right on snoring. Eur J Med Res 1998; 3: 367-379.

11 BaHammam AS, Tate R, Manfreda J, Kryger MH. Upper airway resistance syndrome: effect of nasal dilation, sleep stage, and sleep position. Sleep 1999; 22: 592-598.

12 Gosepath J, Amedee RG, Romantschuck S, Mann WJ. Breathe Right nasal strips and the respiratory disturbance index in sleep related breathing disorders. Am J Rhinol 1999; 13: 385-389.

13 Pevernagie D, Hamans E, Van Cauwenberge P, Pauwels R. External nasal dilation reduces snoring in chronic rhinitis patients: a randomized controlled trial. Eur Respir J 2000; 15: 996-1000.

14 Schonhofer B, Franklin KA, Brunig H, Wehde H, Kohler D. Effect of nasal-valve dilation on obstructive sleep apnea. Chest 2000; 118: 587-590.

15 Wenzel M, Schonhofer B, Siemon K, Kohler D. [Nasal strips without effect on obstructive sleep apnoea and snoring]. Pneumologie 1997; 51: 1108-1110.

16 Hoijer U, Ejnell H, Hedner J, Petruson B, Eng LB. The effects of nasal dilation on snoring and obstructive sleep apnea. Arch Otolaryngol Head Neck Surg 1992; 118: 281-284.

17 Metes A, Cole P, Hoffstein V, Miljeteig H. Nasal airway dilation and obstructed breathing in sleep. Laryngoscope 1992; 102: 1053-1055.

18 Hoffstein V, Mateika S, Metes A. Effect of nasal dilation on snoring and apneas during different stages of sleep. Sleep 1993; 16: 360-365.

19 Braver HM, Block AJ. Effect of nasal spray, positional therapy, and the combination thereof in the asymptomatic snorer. Sleep 1994; 17: 516-521.

20 Kerr P, Millar T, Buckle P, Kryger M. The importance of nasal resistance in obstructive sleep apnea syndrome. J Otolaryngol 1992; 21: 189-195.

21 Craig TJ, Teets S, Lehman EB, Chinchilli VM, Zwillich C. Nasal congestion secondary to allergic rhinitis as a cause of sleep disturbance and daytime fatigue and the response to topical nasal corticosteroids. J Allergy Clin Immunol 1998; 101: 633-637.

22 Brouillette RT, Manoukian JJ, Ducharme FM, et al. Efficacy of fluticasone nasal spray for pediatric obstructive sleep apnea. J Pediatr 2001; 138: 838-844.

23 Dayal VS, Phillipson EA. Nasal surgery in the management of sleep apnea. Ann Otol Rhinol Laryngol 1985; 94: 550-554.

24 Friedman M, Tanyeri H, Lim JW, Landsberg R, Vaidyanathan K, Caldarelli D. Effect of improved nasal breathing on obstructive sleep apnea. Otolaryngol Head Neck Surg 2000; 122: 71-74.
25 Verse T, Maurer JT, Pirsig W. Effect of nasal surgery on sleep-related breathing disorders. Laryngoscope 2002; 112: 64-68.

26 Series F, St Pierre S, Carrier G. Surgical correction of nasal obstruction in the treatment of mild sleep apnoea: importance of cephalometry in predicting outcome. Thorax 1993; 48: 360-363.

27 Sleep-related breathing disorders in adults: recommendations for syndrome definition and measurement techniques in clinical research. The Report of an American Academy of Sleep Medicine Task Force. Sleep 1999; 22: 667-689.

28 Fitzpatrick MF, McLean H, Urton AM, Tan A, O'Donnell DE, Driver HS. Effect of nasal or oral breathing route on upper airway resistance during sleep. Eur Respir $J$ 2003; 22: 827-832.

29 Kamal I. Normal standard curve for acoustic pharyngometry. Otolaryngol Head Neck Surg 2001; 124: 323-330.

30 Fleiss JL. The design and analysis of clinical experiments. New York, Wiley, 1986.

31 Arnedt JT, Wilde GJS, Munt PW, MacLean AW. Simulated driving performance following prolonged wakefulness and alcohol consumption: separate and combined contributions to impairment. J Sleep Res 2000; 9: 233-241.

32 EEG arousals: scoring rules and examples: a preliminary report from the Sleep Disorders Atlas Task Force of the American Sleep Disorders Association. Sleep 1992; 15: 173-184.

33 Rundcrantz H. Postural variations of nasal patency. Acta Otolaryngol 1969; 68: 435-443.

34 Desfonds P, Planes C, Fuhrman C, Foucher A, Raffestin B. Nasal resistance in snorers with or without sleep apnea: effect of posture and nasal ventilation with continuous positive airway pressure. Sleep 1998; 21: 625-632.

35 Lieberman A, Ohki M, Forte V, Fraschetti J, Cole P. Nose/ mouth distribution of respiratory airflow in "mouth breathing" children. Acta Otolaryngol 1990; 109: 454-460.

36 Meurice J-C, Marc I, Carrier G, Series F. Effect of mouth opening on upper airway collapsibility in normal sleeping subjects. Am J Respir Crit Care Med 1996; 153: 255-259.

37 Hollowell DE, Suratt PM. Mandible position and activation of submental and masseter muscles during sleep. J Appl Physiol 1991; 71: 2267-2273.

38 Miyamoto K, Ozbek MM, Lowe AA, et al. Mandibular posture during sleep in healthy adults. Arch Oral Biol 1998; 43: 269-275.

39 Shi YX, Seto-Poon M, Wheatley JR. Breathing route dependence of upper airway muscle activity during hyperpnea. J Appl Physiol 1998; 84: 1701-1706.

40 Basner RC, Simon PM, Schwartzstein RM, Weinberger SE, Weiss JW. Breathing route influences upper airway muscle activity in awake normal adults. J Appl Physiol 1989; 66: 1766-1771.

41 Heimer D, Scharf SM, Lieberman A, Lavie P. Sleep apnea syndrome treated by repair of deviated nasal septum. Chest 1983; 84: 184-185.

42 Verse T, Pirsig W. Impact of impaired nasal breathing on sleep-disordered breathing. Sleep Breath 2003; 7: 63-76.

43 Kamal I. Test-retest validity of acoustic pharyngometry measurements. Otolaryngol Head Neck Surg 2004; 130: 223-228. 